\title{
Los trabajadores sociales en la primera década del siglo XXI. A cuarenta años de la publicación de Situación del servicio social en España, de Jesús María Vázquez
}

\author{
Social workers in the first decade of the $21^{\text {st }}$ cetury. 40 years \\ after Jesús María Vázquez' publicacion Status of social services in Spain
}

\author{
Rogelio GómEz GARcía \\ Universidad de Valladolid \\ rgomga@soc.uva.es \\ Alba TORICES BLANCO \\ Cruz Roja de León \\ atbdts@hotmail.es
}

Recibido: 29/09/2011

Revisado: $15 / 11 / 2011$

Aceptado: 01/03/2012

Disponible on line: 03/08/2012

\begin{abstract}
Resumen
Coincidiendo con el 40 aniversario de la publicación de «Situación del Servicio Social en España», primer estudio sociológico realizado a nivel nacional por Jesús María Vázquez (1971) sobre el Trabajo Social en nuestro país, en este artículo se exponen algunos de los principales cambios que se han producido en nuestra profesión en estas cuatro últimas décadas.
\end{abstract}

Palabras clave: trabajo social, profesión, profesionalización, trabajadores sociales.

\begin{abstract}
Coinciding with the $40^{\text {th }}$ anniversary of the first sociological study on social work in our country, titled «Status of Social Services in Spain» by Jesús María Vázquez (1971), this article presents some of the main changes that have occurred in our profession in the last four decades.

Key words: social work, profession, professionalization, social workers.

Referencia normalizada: Gómez García, R., y Torices Blanco, A. (2012): «Los trabajadores sociales en la primera década del siglo XXI. A cuarenta años de la publicación de Situación del servicio social en España, de Jesús María Vázquez». Cuadernos de Trabajo Social, 25(2): 461-470.
\end{abstract}

Sumario: 1. Punto de partida. 2. Metodología de la investigación. 3. Estructura actual y análisis de resultados. 4. Conclusiones. 5. Bibliografía.

\section{Punto de partida}

En 2011 se cumplió el $40^{\circ}$ aniversario de la publicación de Situación del Servicio Social en España, primer estudio sociológico realizado a nivel nacional por Jesús María Vázquez (1971) sobre el Trabajo Social en nuestro país.

Vázquez era dominico y había seguido cursos de especialización en Sociología y Técnicas de Investigación Social en «Economie et
Humanisme», el célebre centro francés fundado en 1941 por Louis-Joseph Lebret, en Lyon. Doctor en Sociología por la Universidad Pontificia de Salamanca (1966); Doctor en Filosofía y Letras por la Universidad de Madrid (1971); Licenciado en Sociología por la Universidad de Santo Tomás, en Bogotá (1969) se había diplomado en Informática por la Escuela Oficial de Periodismo (1973). Enseñó Técnicas de In- 
vestigación Social, en la Escuela de Formación Social y Familiar (1953-1975), conocida en nuestra profesión como la Escuela de Trabajo Social de Lagasca; así como Sociología de la Religión en el Instituto Social León XIII (1962-1977); desde 1966 a 1978 enseñaba en la Universidad Complutense, pasando a la Universidad de Murcia de 1978 a 1981. Fue catedrático y director del Departamento de Ética y Sociología en la Universidad de Salamanca (1982-1985), así como catedrático de Antropología Social en la Universidad Complutense desde 1985 hasta su jubilación en 1989 (Molina, 1995, pp. 329-331).

Ese estudio se lo encargó el delegado de la Comisión Episcopal de Asistencia Social, Francisco Guijarro Arrizabalaga, como parte de la campaña organizada por la Comisión para «dar a conocer la profesión de Asistente Social, tanto en cuanto a los principios y los métodos que constituyen su contenido como su aplicación al trabajo que realizan en diversos campos de actuación» (Vázquez, 1971, p. 56). Se trata de una obra, de alcance nacional, que identifica los diversos ámbitos del servicio social, los organismos y entidades sociales, las asociaciones y las opiniones de expertos conocedores del Trabajo Social. Se presentaba como una «obra de especial interés para cuantos miran al Servicio Social con ojos inquisitivos o con deseo de comprobar datos concretos, sin resignarse a aceptar vagas referencias» (Vazquez, 1971).

El trabajo de Vázquez fue un referente para otros informes sobre el Trabajo Social: el de Juan Estruch y Antonio M. Güell (1976), sobre la formación del Trabajo Social en Cataluña; el de Juan José Llovet y Ricardo Usieto (1990), sobre los profesionales en la Comunidad de Madrid; el de Molina Sánchez (1994) sobre las enseñanzas de Trabajo Social en España; y el del autor que suscribe este artículo, Rogelio Gómez (2010), sobre los profesionales en la Comunidad de Castilla y León; todos estos estudios hacen referencia obligada a la obra de Vázquez. Cuarenta años después de esta primera publicación presentamos brevemente algunos de los resultados generales de una reciente encuesta de Alba Torices Blanco, que nos permite visionar algunos de los cambios, así como algunas de sus posiciones y respuestas ante los retos del futuro.

\section{Metodología de la investigación}

La recogida de la información se ha realizado mediante una encuesta, diseñada con el programa DYANE Versión 4 (Diseño y análisis de encuestas en investigación social y de mercados) en formato MS Word y enviada por correo electrónico.

El punto de partida para su realización fue el diseño de un cuestionario con el que se elaboró el programa de recogida y tratamiento de la información. Para la selección y redacción de las preguntas referentes a las variables estudiadas, se revisaron otros estudios sobre la evolución y situación de la profesión: Vázquez (1971); Estruch y Güell (1976); Llovet y Usieto (1990); Berasaluze y Berrio-Otxoa (2006); Cuesta Ruiz (2007), Collegi Oficial de diplomats en treball social $\mathrm{i}$ assistents socials de $\mathrm{Ca}$ talunya/Progress (1997) y el autor que suscribe (2010).

El cuestionario elaborado está formado por diferentes tipos de preguntas en función de las posibilidades de respuesta (abiertas y cerradas) y recoge datos de diversa naturaleza (numéricos, de razón, ordinales, textuales, etc.). Se estructura en 6 grupos temáticos: características sociales y demográficas; formación académica; ejercicio profesional; control y satisfacción; profesión y sociedad; organizaciones y profesionales; con un total de 126 preguntas.

El estudio se realizó a escala nacional, el primer paso consistió en enviar una carta de presentación a los/as presidentes/as de los 37 Colegios Oficiales de Diplomados en Trabajo Social y Asistentes Sociales existentes en España, a la que se acompañaba del cuestionario para su estudio y valoración. Si el Colegio decidía participar, debían enviar un correo a todos los colegiados con los que el aquél mantenía contacto a través de correo electrónico, adjuntando el cuestionario y las instrucciones necesarias para responderlo y reenviarlo a una cuenta que se habilitó para recibirlos y resolver cualquier duda (situacion-ts-espana@hotmail.com).

El total de cuestionarios devueltos fue de 505, pero, una vez revisados y descartados los que contenían incorrecciones, el número total se redujo a 485 . Se desconoce el número de colegiados que mantienen contacto con su Colegio a través de correo electrónico, lo cual dificulta el cálculo de la tasa de retorno. La recogida 


\begin{tabular}{|l|c|c|}
\hline \multicolumn{1}{|c|}{ Colegios } & Frecuencia & Porcentaje \% \\
\hline Alicante & 63 & 13 \\
\hline Asturias & 23 & 5 \\
\hline Badajoz & 12 & 2 \\
\hline Burgos & 18 & 4 \\
\hline Cáceres & 17 & 4 \\
\hline Castilla-La Mancha & 22 & 5 \\
\hline Galicia & 146 & 30 \\
\hline Granada & 12 & 2 \\
\hline Guipúzcoa & 18 & 4 \\
\hline León & 30 & 6 \\
\hline Las Palmas & 34 & 7 \\
\hline Navarra & 21 & 4 \\
\hline Sevilla & 42 & 9 \\
\hline Valladolid & 27 & 6 \\
\hline Total frecuencias & 485 & 100 \\
\hline
\end{tabular}

sional. Intentamos, con los datos disponibles, explicar las cuestiones principales que afectan a los trabajadores sociales españoles como profesionales. Los datos que se aportan en este apartado hacen referencia única y exclusivamente a los trabajadores sociales encuestados y en ningún caso se puede inferir a todo el universo.

\subsection{Sociografía de los trabajadores sociales}

Las características sociales y demográficas de la profesión son producto y reflejo de su historia reciente y su posición tanto en la sociedad como en el mercado. Son asimismo elementos clave para entender las formas dominantes de ejercicio, los problemas más acuciantes y las opiniones diferentes de los/as trabajadores/as sociales. La tendencia de entrada en la profesión, la composición de género, la procedencia social, el estatus social alcanzado y su papel dentro de la sociedad son, en gran medida, producto de una demanda y valoración social y organizativa cambiante a la cual la profesión va adaptándose.

Los trabajadores sociales forman un colectivo relativamente joven. La media de edad de los profesionales es de 35 años. La juventud de los trabajadores sociales es el resultado del gran crecimiento que ha experimentado la profesión en las últimas décadas. Si agrupamos los profesionales en franjas de edad, constatamos una articulación caracterizada por:

- El grupo de profesionales de menos de 40 años es el más numeroso de la profesión (75,3 por ciento).

- El grupo de edad entre 41 y 50 años representa el 17,8 por ciento.

- El grupo de más de 51 años es el más reducido $(6,9$ por ciento).

Una de las características persistentes de la profesión es su composición por género: el 90,6 por ciento de los profesionales actuales son mujeres, de las cuales el 46,8 por ciento son católicas. La participación de las mujeres en la profesión apenas ha disminuido a lo largo de los años y siguen constituyendo la fuerza dominante de la profesión. Respecto al estado civil, se puede afirmar que la presencia de casados o que viven en pareja entre los trabajadores sociales es la opción mayoritaria $(50,21$ por ciento). En términos generacionales, los 
profesionales más jóvenes (menos de 40 años) reparten la convivencia entre su cónyuge/pareja $(55,9$ por ciento) y sus padres/amigos $(30,7$ por ciento), mientras que la cohorte de más edad (más de 51 años) convive principalmente con su cónyuge/pareja ( 73,4 por ciento).

La estructura de ingresos de este colectivo está dominada por tres franjas de ingresos mensuales:

- Un primer grupo con ingresos superiores a 1.500 euros mensuales (40,4 por ciento).

- Un segundo grupo con ingresos entre 1.001 y 1.500 euros (39,7 por ciento).

- Un tercer grupo con ingresos inferiores a los 1.000 euros ( 20 por ciento). Dentro de este grupo hay un 4,4 por ciento con ingresos que no llegan a los 600 euros.

Mientras que el 39,7 por ciento de los trabajadores sociales piensan que han ascendido socialmente, un tercio (33,9 por ciento) considera que tiene un estatus social similar al de los padres y el 26,4 por ciento piensa que ha bajado. Vemos como la profesión deja de ser un mecanismo de ascenso social y, en cambio, aumenta progresivamente el descenso social. De hecho, el 38,3 por ciento de los trabajadores sociales piensan que su posición actual es más baja que la que esperaba lograr cuando entró en la profesión. El 42,4 por ciento considera que ha logrado el que esperaba y para el 19,3 por ciento su posición actual es superior a la que esperaba.

Los trabajadores sociales se sitúan en una posición ideológica media de izquierda $(3,6$ en una escala de 0 como izquierda a 10 como derecha), de hecho más a la izquierda que la media poblacional que se sitúa, según el CIS en 4,93 (CIS, 2011, p. 12). Esa posición ideológica les acerca a partidos como: el Partido Socialista Obrero Español (25,4 por ciento) e Izquierda Unida (10,9 por ciento). Llama la atención que el 25,4 por ciento considera que ningún partido representa su ideología y un 22,9 por ciento no ha contestado.

La familia (9,54 sobre 10), los amigos $(8,59$ sobre 10$)$ y el trabajo $(8,05$ sobre 10$)$ son, en este orden, los elementos más importantes de este colectivo. Tanto en la familia como en las relaciones sociales la profesión desempeña un papel muy destacado: el 50,0 por ciento de las amistades son de la misma profesión y casi el
20,0 por ciento de los parientes pertenecen también a la profesión $(19,59$ por ciento son hermano y 14,43 por ciento cónyuge). Además, en cuanto a la variable formativa, el 24,8 por ciento de los profesionales encuestados tiene otra titulación universitaria (diplomatura, licenciatura, doctorado).

\subsection{La práctica profesional de los trabaja- dores sociales}

El 80 por ciento de los profesionales ejercen su profesión y el 4,6\% aunque trabaja, no desempeña la labor como trabajador social. Casi tres cuartas partes ( 72 por ciento) de estos profesionales realizan su actividad como empleados estables (contrato indefinido). En el otro extremo nos encontramos con el 27,9 por ciento de los profesionales que ocupan unas posiciones laborales precarias (con contratos temporales). El 85,8 por ciento de los trabajadores sociales ejercen su actividad profesional con una dedicación de jornada completa y en un 14,2 por ciento de los casos a jornada parcial. La media del tiempo que llevan ejerciendo como trabajadores sociales es de 10 años, con una desviación típica de 8 años. Estos profesionales desarrollan su actividad principalmente en el sistema público de servicios sociales (Administración central, autonómica, provincial y local; 58,4 por ciento), en el Tercer sector $(29,9$ por ciento) y en el sector lucrativo (11,7 por ciento).

Los sectores de población objeto de intervención social más relevantes son: el 20,7 por ciento atienden al conjunto de población, el 15,4 por ciento trabajan con personas mayores, el 14,0 por ciento con personas que padecen algún tipo de discapacidad, el 11,2 por ciento en el sector salud y el 9,5 por ciento con personas en situación de exclusión social. En los restantes sectores de intervención su presencia se menor.

Sobre las tareas propias de la intervención directa del Trabajo Social se señala que más del 57 por ciento de las profesionales desarrollan cada una de las tareas indicadas (entrevistas, el 84,36 por ciento; información, orientación y derivación, el 85,31 por ciento; atención telefónica, el 72,51 por ciento; visitas domiciliarias, el 57,11 por ciento; valoración diagnóstica, el 68,96 por ciento; elaboración de planes de intervención, el 57,11 por ciento y contacto 
con el entorno social, el 57,82 por ciento), a excepción de las sesiones de grupo que las realizan el 24,88 por ciento.

Al igual que con las tareas de intervención directa, con las de intervención indirecta también se han seleccionado las más relevantes y las tareas ampliamente realizadas son: reuniones con otros profesionales ( 86,71 por ciento), tareas administrativas $(82,28$ por ciento $)$ y elaboración de informes, fichas o historias sociales $(81,82$ por ciento). Por el contrario, las tareas menos habituales son la participación en estudios o investigaciones ( 18,18 por ciento) y la organización de campañas de prevención (24,71 por ciento).

Las funciones desempeñadas por la mayoría de los trabajadores sociales son: información y orientación, el 86,71 por ciento; tareas administrativas, el 76,46 por ciento; planificación, el 59, 21 por ciento y asistencia, el 53,85 por ciento.

Los trabajadores sociales en su ejercicio profesional dedican por término medio el siguiente tiempo a los diferentes tipos de intervención: a la intervención individual y familiar, entre el 50 por ciento y el 60 por ciento de su tiempo; a la intervención colectiva, entre el 20 por ciento y el 30 por ciento del mismo; a la organización-documentación, dedican entre el 40 por ciento y el 50 por ciento de su tiempo; a la programación y planificación de intervenciones directas, entre el 30 por ciento y el 40 por ciento; a intervenciones en el entorno del asistido, el tiempo dedicado es entre el 30 por ciento y el 40 por ciento; y a intervenciones en organismos sociales, entre el 30 por ciento y el 40 por ciento de su tiempo

La mayoría de los trabajadores sociales desarrollan siempre $(25,29$ por ciento) o con mucha frecuencia $(35,27$ por ciento) su actividad laboral en coordinación con otros profesionales, siendo principalmente con otros trabajadores sociales $(80,19$ por ciento) seguido de psicólogos $(59,44$ por ciento) y personal de enfermería $(28,90$ por ciento).

\subsection{Control y satisfacción laboral de los tra- bajadores sociales}

Uno de los temas centrales de toda profesión son sus niveles de control y autonomía profesional, nos indican su grado de profesionalización. Al analizar esta dimensión nos centramos en tres tipos de control básico: control técnico, control organizativo y control político. El primero se centra en lo que podríamos llamar el corazón de toda profesión, en su conocimiento, y hasta qué punto social y organizativamente se valora el conocimiento profesional como suficiente para la toma de decisiones técnicas independiente. El segundo gira en torno a la organización social del trabajo y mide la capacidad profesional de aplicar la racionalidad organizativa y de realizar del trabajo. El tercero tiene que ver con la influencia de una profesión en la orientación política de la organización en la que trabaja y en qué medida los objetivos profesionales llegan a ser parte dominante o no de los objetivos organizativos.

Las áreas en que los profesionales consideran que tienen un nivel más alto de control se relacionan con el proceso de atención: consultas con otros colegas $(6,93$ de media) y tiempo dedicado a cada persona atendida $(6,72$ de media). Tienen menos control (aun cuando continúa siendo alto) sobre lo que configura el núcleo del ejercicio profesional: las técnicas o procedimientos a aplicar en su trabajo $(6,83 \mathrm{de}$ media). El nivel de control sobre la organización social y política del trabajo es bajo: horario de trabajo $(5,95$ de media), diseño de las prioridades y objetivos de la organización $(5,22$ de media) y composición del equipo con quien trabaja (4,54 de media).

Su imagen pública divide a la profesión casi por la mitad. Algo más de la mitad de los profesionales $(57,1$ por ciento) piensan que la opinión pública tiene una buena imagen de ellos y el 42,9 por ciento piensa que no. Los trabajadores sociales se sienten bien valorados por los usuarios (86,5 por ciento) y por los colegas ( 88 por ciento) $\mathrm{y}$, en menor medida por las propias organizaciones $(69,4$ por ciento).

Un gran número de trabajadores sociales (41,5 por ciento) están insatisfechos con su estatus social, es decir, con la forma en que son valorados socialmente. Sólo algo más de la cuarta parte $(34,2$ por ciento) de los trabajadores sociales están satisfechos y el 24,4 por ciento restante parecen estar resignados (no están satisfechos ni insatisfechos). Los profesionales tienen la sensación de que su profesionalidad y su contribución no son lo suficiente valoradas ni compensadas por la sociedad. Esto hace que no todos tengan la completa certe- 
za de que volverían a entrar en la profesión. El 64,5 por ciento no tendría la menor duda en volver a la profesión y el 25,6 por ciento, en cambio, tendría que pensárselo. El 7,8 por ciento de los profesionales tiene muchas dudas y el 2,1 por ciento no volvería a entrar en la profesión.

Las problemáticas que impiden que el nivel de satisfacción sea más alto y que causen las dudas respecto a la profesión son las siguientes: las retribuciones insuficientes $(7,58)$; la falta de incentivos profesionales $(7,7)$; la precariedad laboral $(7,87)$; la falta de reconocimiento social y político $(7,56)$; la dificultad debida a que las prioridades profesionales sean parte de los objetivos organizativos $(7,39)$; las expectativas profesionales no satisfechas $(7,22)$; la inadecuación entre formación y trabajo actual $(7,15)$; las dificultades de acceso a la formación continua $(6,76)$ y la rutina del ejercicio profesional $(7,09)$.

Los problemas más importantes reflejan la insatisfacción por la manera en que se valora su trabajo y dedicación (retribuciones insuficientes), por la carencia de posibilidades de realización de un ejercicio (falta de incentivos, precariedad profesional) y la insatisfacción por la disonancia entre sus expectativas profesionales y la realidad cotidiana. La gran mayoría (87,5 por ciento) aspira a asumir mayores niveles de responsabilidad en la toma de decisiones en su trabajo y se considera capacitado para ello.

El ejercicio actual de los trabajadores sociales contiene algunos de los elementos centrales que garantizan la satisfacción con la profesión. Lo más importantes es que su trabajo contribuye a mejorar la calidad de vida de las personas $(83,91$ por ciento). En un trabajo ideal, los elementos que destacan son precisamente algunos de los que más echan en falta en su trabajo actual: que esté retribuido adecuadamente $(74,89$ por ciento).

\subsection{Sociedad y trabajadores sociales}

El elemento social es fundamental en la definición y en el ejercicio del Trabajo Social. La orientación social de la profesión caracteriza tanto su papel y los valores sociales, como su sistema de excelencia y sus perspectivas de futuro. El prestigio adquirido por las profesiones dentro de la estructura social, es un proceso que comienza en el siglo XIX con el establecimiento de un nuevo concepto de profesionalismo basado en un aprendizaje específico y unas responsabilidades bien definidas (Elliot, 1974 citado en Ortega y Humanes (2000, p. 159). La ocupación se convirtió, para los sociólogos funcionalistas, en uno de los indicadores más claros para medir la posición dentro de la estructura social. Entre los profesionales domina la insatisfacción con respecto a su estatus social que refleja un malestar fundamental anteriormente señalado, por no sentirse valoradas los suficiente por la sociedad.

Las fuentes de prestigio muestran lo que debería ser importante y central en la profesión. Algunos de los valores principales futuros señalados por las trabajadoras sociales como esencia de prestigio son: la ética e integridad profesional (9,21 de media); el éxito en la solución de los casos (7,97 de media), la ayuda a colegas $(7,88$ de media), las ideas innovadoras (7,86 de media) y la alta dedicación a la profesión (7,47 de media).

Las profesionales definen su papel orientado al bienestar (bienestar de la sociedad, 36,8 por ciento; bienestar de los usuarios 21,6 por ciento) y satisfacción de las necesidades de la sociedad en su conjunto (satisfacer las necesidades de los usuarios, 21 por ciento; satisfacer las necesidades de la población, 12,7 por ciento): prima, como se observa, la orientación del bienestar colectivo por encima de la orientación individual.

Consideran que las personas a las que atienden esperan principalmente del trabajador social que le garantice sus derechos ante el sistema de servicios sociales $(26,24$ por ciento) y que contribuya a solucionar sus problemas $(25,30$ por ciento). Para la mayoría de los encuestados, la profesión consiste principalmente en una forma de ayudar a los demás (7,38 de media), una fuente de satisfacción (7,23 de media) y un trabajo (7,15 de media). La visión altruista domina sobre la visión laboral o profesional que naturalmente también son importantes. En cambio, no se considera como una forma de vida (6,25 de media), ni lo más importante de su vida (4,38 de media) y ni siquiera una forma de ascenso social o económica $(4,88$ de media).

Los trabajadores sociales dedican por término medio el siguiente tiempo a: la práctica 
profesional ( 7,18 horas); el cuidado y atención de los hijos (4,40 horas); al cuidado del hogar (2,36 horas); al ocio ( 2,43 horas) las relaciones sociales (2,44 horas); el estudio (2,41 horas), el cuidado de familiares mayores o dependientes (1,82 horas); las actividades sociales y de voluntariado (1,97 horas) y otras actividades laborales (2,21 horas). Consideran además que pueden compatibilizar bastante bien $(5,34$ de media sobre 7) su vida laboral y familiar.

\subsection{Organización profesional de los traba- jadores sociales}

Veamos aquí la visión que tienen los trabajadores sociales de los Colegios profesionales oficiales. La valoración que los profesionales realizan de la utilidad del Colegio es un 6,19 sobre 10 y su nivel de representatividad es un 5,90 sobre 10 . Los trabajadores sociales valoran las funciones de los Colegios Oficiales de la siguiente forma: dar una cobertura suficiente a la responsabilidad civil de los trabajadores sociales (6,55 de media); llevar a cabo cursos de formación (6,47 de media); estar presente en el debate social (6,52 de media); defender los intereses profesionales ante las administraciones públicas (6,59 de media); posicionarse ante la sociedad sobre temas sociales $(6,42 \mathrm{de}$ media); ayudar a los trabajadores sociales $(6,70$ de media); controlar la praxis del Trabajo Social $(6,16$ de media); ejercer funciones disciplinarias y sancionar las faltas de los profesionales (5,29 de media); atender a sociedad y temas de interés social $(6,07$ de media)

\section{Conclusiones}

El resumen de las principales características, opiniones y valores expresados por los profesionales en la encuesta dibujan una clara imagen de las características de los trabajadores sociales en esta primera década del siglo XXI: son profesionales jóvenes (edad media de 35 años), en su mayoría mujeres $(90,6$ por ciento) de religión católica (46,8 por ciento); el 50,21 por ciento están casados o viven en pareja; con unos ingresos mensuales por encima de los 1.500 euros (40 por ciento) y un 20 por ciento con ingresos inferiores a 1.000 euros mensuales; algo más de la tercera parte $(38,3$ por ciento) de los profesionales no han logrado todavía la posición social que esperaban cuando entraron en la profesión; se trata de un colectivo ideológicamente de iz- quierdas (3,6 en una escala de 0 como extrema izquierda a 10 como extrema derecha), que vota principalmente al Partido Socialista Obrero Español (25,4 por ciento) e Izquierda Unida (10,9 por ciento); una cuarta parte de los profesionales $(24,8$ por ciento) tiene otra titulación universitaria; el 80 por ciento ejerce su actividad dentro de la profesión; la mayoría (72 por ciento) tiene una relación contractual estable, pero existe casi un 28 por ciento que está en una situación contractual precaria; el tipo de contrato con el que ejercen su actividad profesional es a jornada completa $(85,8$ por ciento) y la media del tiempo que llevan ejerciendo son 10 años; las formas dominantes de ejercicio caracterizan el trabajo social como una profesión mayoritariamente empleada en el sector público $(58,5$ por ciento) con una importante presencia en el Tercer sector $(29,9$ por ciento). Una quinta parte de los profesionales (20,7 por ciento) tiene al conjunto de la población como principal sector de intervención.

Las tareas de intervención directa más realizadas son: la entrevista, la información, orientación-derivación y la atención telefónica. Dentro de las tareas de intervención indirecta destacan: las reuniones con otros profesionales, las tareas administrativas y la elaboración de informes, fichas o historias sociales. Las funciones más desarrolladas son la informativa y las tareas administrativas. Desarrollan siempre (25,29 por ciento) o con mucha frecuencia $(35,27$ por ciento) $\mathrm{su}$ actividad laboral en coordinación con otros profesionales, principalmente trabajadores sociales (80,19 por ciento y psicólogos $(59,44$ por ciento). En términos globales tienen un alto nivel de control y autonomía y consideran que la opinión pública tiene una buena imagen de ellos $(57,1$ por ciento), además se sienten bien valorados por los usuarios (86,5 por ciento) y los colegas (88 por ciento) pero insuficientemente valorados por la organización $(69,4$ por ciento) y la sociedad (57,1 por ciento). En general (41,5 por ciento) se sienten insatisfechos con su estatus social, si bien la mayoría $(64,5$ por ciento) volvería a entrar.

Los problemas más importantes en su ejercicio son la precariedad laboral $(7,87$ de media) la falta de incentivos profesionales $(7,7 \mathrm{de}$ media), las retribuciones insuficientes $(7,58 \mathrm{de}$ media) y la falta de reconocimiento social y político (7,56 de media). 
La gran mayoría ( 87,5 por ciento) aspira a asumir mayores niveles de responsabilidad y también para la mayoría de los profesionales, su profesión consiste fundamentalmente en una forma de ayudar a los demás, siendo su labor estar al servicio del bienestar de las personas. Asimismo, consideran que pueden compatibilizar bastante bien $(5,34$ de media sobre 7$) \mathrm{su}$ vida laboral y familiar.

La valoración que los profesionales realizan de la utilidad de su Colegio Profesional es de 6,19 sobre 10. Las funciones de los Colegios Oficiales mejor valoradas son la ayuda a los profesionales $(6,70$ sobre 10$)$, la defensa de los intereses profesionales ante las administraciones públicas $(6,59$ sobre 10$)$ y el dar cobertura suficiente a la responsabilidad civil de los profesionales $(6,55$ sobre 10$)$.

Como podemos observar, los cambios que han acaecido en la profesión en estas cuatro últimas décadas han sido profundos, entre los que cabe destacar:

\subsection{El perfil de los trabajadores sociales ha cambiado}

Desde los años setenta hasta ahora el perfil de los trabajadores sociales ha cambiado. Dos son las grandes líneas de influencia que han hecho posible este cambio: por un lado, las importantes transformaciones que se han producido en el entorno social, económico y político del país durante las últimas cuatro décadas. En segundo lugar, la influencia de la consideración universitaria del Trabajo Social para la profesión, y especialmente para el perfil social de los trabajadores sociales.

Tradicionalmente, el Trabajo Social era una profesión realizada por mujeres. De hecho no ha sido hasta los últimos años cuando, progresivamente, se han ido incorporando a la misma los varones, y como hemos visto en el transcurso de la investigación, el porcentaje de hombres en la profesión ha pasado de ser nulo en el año 1971 al 9,4 por ciento en la actualidad. El análisis por edades indica que sigue siendo una profesión constituida en su mayoría por personas jóvenes. Los profesionales menores de cuarenta años ha pasado del 80 por ciento al 75,3 por ciento en la actualidad. En relación con el estado civil se ha descendido del 72 por ciento de profesionales solteras al 44,42 por ciento actual.

\subsection{La situación laboral de los trabajado- res sociales ha cambiado}

Las características de la contratación laboral de los trabajadores sociales han experimentado un fuerte y positivo adelanto en los últimos cuarenta años. De asociarse muchas veces a prácticas voluntarias se ha pasado al reconocimiento profesional que denota la contratación de profesionales con unas tareas específicas y especializadas que las hacen diferentes del resto de prácticas profesionales.

En España en 1970, la situación de los trabajadores sociales rozaba el escándalo. El 53 por ciento de los profesionales no tenía contrato de trabajo, un dato que era, por una parte, signo de debilidad estructural de la profesión, y por la otra, indicio de una ocupación a la que precisamente no terminaba de conferírsele el rango de profesional. Además un 30 por ciento de las personas que trabajaban sin contrato no lo habían solicitado e incluso había muchas trabajadores que declaraban no considerarlo necesario (Vázquez, 1971, pp. 187188). En la actualidad la precariedad laboral de los trabajadores sociales es escasa y tener a las administraciones públicas como el principal contratista asegura una estabilidad laboral continua. En el momento actual casi el 60 por ciento de los trabajadores sociales desarrollan su actividad dentro de la administración. En el trabajo de Vázquez, de cada cien titulados sólo ejercían la profesión sesenta y tres, mientras que actualmente son ya ochenta los ocupados.

\subsection{La problemática profesional de los tra- bajadores sociales ha cambiado}

Se aprecia como algunas de las problemáticas profesionales continúan (las retribuciones insuficientes, la realización de tareas administrativas y burocráticas, la falta de reconocimiento social y político, etc.), y otras se han alterado (la falta de preparación profesional, el paro profesional, etc.). Entre los trabajadores sociales hay una importante presencia de juicios críticos en torno a la calidad del ejercicio profesional. Mientras que en 1971 el 64 por ciento de los trabajadores sociales manifestaban encontrarse satisfecho profesionalmente, en la actualidad ese porcentaje se ha reducido casi a la mitad $(33,3$ por ciento). 


\subsection{La formación de los trabajadores socia- les ha cambiado}

El ejercicio profesional es, sin duda, una fuente de adquisición de nuevas habilidades y conocimientos (formación más específica), pero también es un elemento que decanta como aprendizaje profesional la formación recibida. Los estudios de Trabajo Social han pasado por una serie de etapas muy diferenciadas: desde la formación sin reconocimiento oficial por parte del Ministerio de Educación (hasta 1964), pasando por una formación con reconocimiento oficial de grado medio no universitario (hasta 1980), hasta su primer reconocimiento como estudios universitarios de primer ciclo (hasta 2008) y finalmente, su consagración como estudios de Grado en Trabajo Social (a partir de 2009), como consecuencia de la implantación del Espacio Europeo de Educación Superior

Deben ser pocas las profesiones que no otorguen gran importancia al ejercicio profesional en los procesos de aprendizaje. Sin embargo, siempre que puedan, pondrán en valor la formación teórica recibida (se trata de un capital que se valora tanto más cuanto más difícil es de adquirir). Estas consideraciones nos permiten comprender que el juicio sobre cierta debilidad formativa realizado por profesionales con experiencia es creíble y es probablemente por ello mismo por lo que que dar continuidad a la formación a lo largo de la vida profesional no es cosa de minorías: mientras que en el estudio de Vázquez la proporción de quienes decían tener estudios superiores era el 5 por ciento, en la presente investigación el 25 por ciento de los entrevistados declara haber realizado otra carrera.

\subsection{Las áreas de Trabajo Social en que los trabajadores sociales ejercen ha cambiado} Por su idiosincrasia, el Trabajo Social se orienta a una multiplicidad de ámbitos y la cuestión de cuál es la clasificación más idónea no es nueva. Ya en el trabajo de Vázquez se decía que la «clasificación en sectores (Trabajo, Enseñanza, Sanidad, Vivienda y Comunidad Social) no es excluyente ni exhaustiva en su enunciación» y se subrayaba «la carencia de una tipología definitiva y adaptada a nuestro contexto sociocultural» (Vázquez, 1971, p. 139).

Los porcentajes de profesionales en ejercicio, según los campos sectoriales de Servicio Social eran, según Vázquez (pp. 302-303) los siguientes: el sector trabajo, el 22,01 por ciento; el sector enseñanza, el 2,92 por ciento; el sector sanidad, el 19,53 por ciento; el sector vivienda el 0,82 por ciento; y el sector comunidad social, el 36,72 por ciento.

En esta investigación hemos considerado conveniente deslindar la nueva realidad que al menos como concepción ordenadora son los Servicios Sociales y aquellas áreas que, formando parte del Bienestar Social, no son cubiertas por los Servicios Sociales. Realizada esta operación, la clasificación sobre las áreas queda como sigue: de población en general, el 19,3 por ciento; de infancia, el 1,6 por ciento; de juventud, el 2,3 por ciento; de familia, el 4,9 por ciento; de personas mayores, el 14,8 por ciento; de personas drogodependientes, el 3,2 por ciento; de inmigrantes, el 1,4 por ciento; de mujer, el 3,2 por ciento; de empleo, el 0,9 por ciento; de personas con discapacidad, el 14,2 por ciento; de otras minorías étnicas, el 1,6 por ciento; de salud, el 10 por ciento; de educación, el 3,9 por ciento; de exclusión social, el 10 por ciento; de; reclusos, el 2,3 por ciento; y de otros sectores, el 6,3 por ciento.

En la primera categoría (Servicios Sociales generales o población en general) están los trabajadores sociales que no orientan su actividad a un sector de población y a un ámbito concretos y constituyen la principal área para una quinta parte de los profesionales; le siguen los diferentes sectores (Servicios Sociales especializados) que representan el 80 por ciento y entre ellos, destacan personas mayores $(14,8$ por ciento) y personas con discapacidad ( 14,2 por ciento).

En este aspecto la comparación con el estudio realizado por Vázquez resulta poco ilustradora, ya que los indicadores utilizados son diferentes han empleado en el presente trabajo. El contacto y la interacción personal con la población sigue siendo el corazón de las tareas del trabajador social. En España en 1970 las visitas, gestiones y desplazamientos eran las funciones a las que dedicaban la mayor parte del tiempo (p. 194).

\subsection{El futuro de los trabajadores sociales ha cambiado}

Los últimos cuarenta años han sido especialmente importantes para el Trabajo Social puesto que la esfera de la protección social ha ex- 
perimentado un notorio crecimiento en nuestro país. Actualmente el futuro del Estado del Bienestar origina intensos y amplios debates en España, Europa y en el mundo. La polémica se centra en encontrar, si existen, los mecanismos para continuar manteniendo el creci- miento teóricamente ilimitado de la protección social con las adecuaciones presupuestarias pertinentes. El mantenimiento de la política de convergencia con Europa es uno de los factores que parece jugar a favor del desarrollo de la protección social.

\section{Referencias bibliográficas}

Barbero, J. M. (2002). El trabajo social en España. Zaragoza: Mira Editores.

Berasaluze, A. y Berrio-otxoa, K. (2006). El ejercicio profesional del Trabajo Social Hoy. Vitoria-Gasteiz: Colegios Oficiales de Diplomados/as en Trabajo Social de Araba, Bizkaia y Guipuzkoa.

Collegi oficial de Diplomats en Treball social i Assistents socials de Catalunya/Progress (1997). Els Diplomats en treball social $i$ assistents socials de Catalunya: perfil i expectativas professionals. Barcelona: Editorial Hacer.

Consejo General de Colegios oficiales de Diplomados en Trabajo Social y Asistentes sociales (1999). Código Deontológico de la Profesión de Diplomado en Trabajo Social.

Cuesta Ruiz, A. B. (coord.) (2007). Situación laboral de las Trabajadoras Sociales en La Rioja. Nuevos retos y propuestas de futuro. Logroño: Colegio Oficial de Diplomados en Trabajo Social y Asistentes Sociales de La Rioja.

Elliot, P. (1974). Sociología de las profesiones. Madrid: Tecnos.

Estruch, J. y Güell, A. (1976). Sociología de una profesión. Los asistentes sociales. Barcelona: Península.

Gómez García, R. (2010). El Trabajo social en Castilla y León: Estudio sociológico de una profesión. León: Universidad de León, Área de publicaciones.

Gómez García, R (2010). Situación del Trabajo Social en Castilla y León. Cuadernos de Trabajo Social, 23, 211-227.

Las Heras, P. y Cortajarena, E. (1979). Introducción al Bienestar social. Madrid: FEDAS.

Llovet, J. J. y Usieto, R. (1990). Los trabajadores sociales. De la crisis de identidad a la profesionalización. Madrid: Popular.

Molina Sánchez, Mª V. (1995). Efemérides. En el recuerdo: Jesús Ma Vázquez Rodríguez (19231995), Catedrático de Sociología. Cuadernos de Trabajo Social, 8, 329-331.

Ortega, F. y Humanes, Mª L. (2000). Algo más que periodistas. Sociología de una profesión. Barcelona: Ariel Sociológica.

Red, N. de la y Brezmes, M. (2003). Trabajo social en España. En T. Fernández y C. Alemán (dirs), Introducción al trabajo social. Madrid: Alianza Editorial.

Rubiol, G. (1973). Investigación en Trabajo Social. En Memoria del II Congreso Nacional de Asistentes Sociales (pp. 86-87). Madrid: Ed. Federación Española de Asistentes Sociales.

Vázquez, J. Ma. (dir.) (1971). Situación del Servicio Social en España. Madrid: Instituto de Sociología Aplicada. 